\title{
External Dynamical Equivalence of Analytic Control Systems
}

\author{
Zbigniew Bartosiewicz*, Ewa Pawłuszewicz ${ }^{\dagger}$ \\ Bialystok Technical University, Faculty of Computer Sciences, Poland \\ baros@pb.edu.pl, epaw@pb.edu.pl
}

\begin{abstract}
Summary. Theory of systems on homogeneous time scales unifies theories of continuous-time and discrete-time systems. The characterizations of external dynamical equivalence known for continuous-time and discrete-time systems are extended here to systems on time scales. Under assumption of uniform observability, it is shown that two analytic control systems with output are externally dynamically equivalent if and only if their delta universes are isomorphic. The delta operator associated to the system on a time scale is a generalization of the differential operator associated to a continuous-time system and of the difference operator associated to a discrete-time system.
\end{abstract}

\section{Introduction}

In 1988, in his Ph.D. thesis [13], Stefan Hilger developed calculus on time scales, which unified the standard differential calculus and the calculus of finite differences. This allowed for a unified treatment of dynamical systems with continuous and discrete time. The book by M. Bohner and A. Peterson [7] contains the most important achievements in this area. But the theory is much richer than just the unification. One can study systems for which time is partly continuous and partly discrete. Many systems appearing in engineering, biology and economy exhibit such features.

In the classical control theory there always have been two parallel areas of research: the continuous-time and the discrete-time systems. Most of the results are similar for both classes of systems, but there are also significant differences. For example, asymptotic stability of linear time-invariant systems is characterized by the condition that the eigenvalues of the system lie in the specific region of the complex plane. However this region depends on the class of systems. Another difference concerns solutions of the differential equations (continuous time) and the difference equations (discrete time). Under some

\footnotetext{
* Supported by Bialystok Technical University grant No W/WI/1/07

$\dagger$ Supported by Bialystok Technical University grant No W/WI/18/07
} 
reasonable conditions ordinary differential equations can be solved forward and backward, while difference equations usually can be solved only forward and some extra assumptions are required to solve them backward. Fortunately, in control theory we are mostly interested in forward solutions, so this difference is less important.

Calculus on time scales entered control theory just a few years ago. First results concerned basic properties of linear systems, like controllability, observability and realizations (see $[4,9,6]$ ). In [5] we studied dynamical feedback equivalence of nonlinear systems on time scales. The main result of that paper will be used here to show a characterization of external dynamical equivalence for systems on time scales. Another attempt to unify continuous-time and discrete-time systems, without use of calculus on time scales, was made in $[11,18]$.

Dynamic equivalence for nonlinear continuous-time systems was first studied by B. Jakubczyk $[14,15]$. He used a dynamical state feedback to transfer trajectories of one system onto trajectories of the other. His concept of dynamical feedback linearizability was close to the property of flatness introduced earlier by M. Fliess (see e.g. [10,17] and [22]). The main result of [14] says that two systems are dynamically (state) feedback equivalent if and only if their differential algebras are isomorphic. In [2] dynamical state feedback equivalence of discrete-time systems was studied. In the characterization of this property, difference algebras were used instead of differential algebras. This was one of the examples where the results for continuous time and discrete time are close to each other and a clever change of language is enough to switch between two classes of systems.

In $[20,3]$ external dynamical equivalence and linearization for discretetime systems were studied. The systems were equipped with output parts and dynamical output feedback was used instead of dynamical state feedback. Necessary and sufficient criteria of external dynamical equivalence were expressed with the aid of the output difference universe. The concept of (function) universe, introduced by J. Johnson, is a generalization of the notion of (function) algebra $[1,16]$. Besides standard algebraic operations possible in algebras, the structure of universe allows for substituting elements of the universe (which are partially defined functions) into real-analytic functions of several variables and amalgamation of partially defined functions from the universe. The results of $[20,3]$ were then transferred (back) to continuous-time systems, with the output differential universe as the key tool [21].

In [5] we studied dynamical state feedback equivalence for nonlinear systems on homogeneous time scales. The results unified those obtained for continuous-time and discrete-time systems. Instead of differential and difference operators used in earlier works, we introduced so called delta operator, which for a particular time scale would become either a differential operator or a difference one.

In this paper we complete the picture studying external dynamical equivalence for control systems with outputs, defined on homogeneous time scales. 
The main result says that two systems are externally dynamically equivalent if and only if their delta universes are isomorphic. This theorem may be seen as an extension of the result of [5] to systems with output or as a unification of our earlier results from [20] and [21]. We assume that the systems are uniformly observable and use the main theorem of [5] to prove the present version. In [3] and [21] we showed that for continuous-time and discrete-time cases one can drop the observability assumptions. Only some regularity of the space obtained by gluing up indistinguishable states of the system is assumed. This suggests that the same could be done for systems on time scales.

The paper is organized as follows. In Section 2 we provide the reader with the necessary background on the calculus on time scales. Section 3 contains setting of the problem and the precise definition of external dynamical equivalence. In Section 4 we recall basic concepts of the theory of universes. The main result of the paper is stated and proved in Section 5 .

\section{Calculus on time scales}

We recall here basic concepts and facts of the calculus on time scales. For more information the reader is referred to [7].

A time scale $\mathbb{T}$ is an arbitrary nonempty closed subset of the set of real numbers $\mathbb{R}$. The standard examples of time scales are $\mathbb{R}, h \mathbb{Z}, h>0, \mathbb{N}, \mathbb{N}_{0}$, $2^{\mathbb{N}_{0}}$ or $\mathbb{P}_{a, b}=\bigcup_{k=0}^{\infty}[k(a+b), k(a+b)+a]$. The time scales $\mathbb{T}$ is a topological space with the relative topology induced from $\mathbb{R}$.

The following operators on $\mathbb{T}$ are often used:

- the forward jump operator $\sigma: \mathbb{T} \rightarrow \mathbb{T}$, defined by $\sigma(t):=\inf \{s \in \mathbb{T}: s>t\}$ and $\sigma(\sup \mathbb{T})=\sup \mathbb{T}$, if $\sup \mathbb{T} \in \mathbb{T}$,

- the backward jump operator $\rho: \mathbb{T} \rightarrow \mathbb{T}$, defined by $\rho(t):=\sup \{s \in \mathbb{T}: s<$ $t\}$ and $\rho(\inf \mathbb{T})=\inf \mathbb{T}$, if $\inf \mathbb{T} \in \mathbb{T}$,

- the graininess functions $\mu, \nu: \mathbb{T} \rightarrow[0, \infty)$ defined by $\mu(t):=\sigma(t)-t$ and respectively by $\nu(t):=t-\rho(t)$.

Points from the time scale can be classified as follows: a point $t \in \mathbb{T}$ is called

- right-scattered if $\sigma(t)>t$ and right-dense if $\sigma(t)=t$,

- left-scattered if $\rho(t)<t$ and left-dense if $\rho(t)=t$,

- isolated if it is both left-scattered and right-scattered,

- dense if it is both left-dense and right-dense.

We define also the sets

$$
\mathbb{T}^{\kappa}:= \begin{cases}\mathbb{T} \backslash\{M\}, & \text { if } M \text { is the left scattered maximum of } \mathbb{T} \\ \mathbb{T}, & \text { if } \sup \mathbb{T}=\infty .\end{cases}
$$




$$
\mathbb{T}_{\kappa}:= \begin{cases}\mathbb{T} \backslash\{m\}, & \text { if } m \text { is the right scattered minimum of } \mathbb{T} \\ \mathbb{T}, & \text { otherwise. }\end{cases}
$$

Example 1. If $\mathbb{T}=\mathbb{R}$ then $\rho(t)=t=\sigma(t)$ and $\mu(t)=\nu(t)=0$, for all $t \in \mathbb{R}$.

Example 2. If $\mathbb{T}=h \mathbb{Z}, h>0$, then $\rho(t)=t-h, \sigma(t)=t+h$, and $\mu(t)=$ $\nu(t)=h$, for all $t \in h \mathbb{Z}$.

Example 3. If $\mathbb{T}=\overline{q^{\mathbb{Z}}}:=\left\{q^{k}: k \in \mathbb{Z}\right\} \cup\{0\}$, where $q>1$, then $\rho(t)=\frac{t}{q}$, $\sigma(t)=q t, \nu(t)=\frac{t(q-1)}{q}$ and $\mu(t)=(q-1) t$, for all $t \in \mathbb{T}$.

Definition 1. A time scale $\mathbb{T}$ is called homogeneous if $\mu$ and $\nu$ are constant on respectively $\mathbb{T}^{\kappa}$ and $\mathbb{T}_{\kappa}$.

The time scales $\mathbb{R}, h \mathbb{Z},[0,1]$ are homogeneous, whereas $\overline{q^{\mathbb{Z}}}$ is not. In this paper we will be interested in homogeneous time scales. Thus we rather want to unify the continuous-time and discrete-time cases, and not to develop a general theory.

Definition 2. Let $f: \mathbb{T} \rightarrow \mathbb{R}$ and $t \in \mathbb{T}^{k}$. Then the number $f^{\Delta}(t)$ (when it exists), with the property that, for any $\varepsilon>0$, there exists a neighborhood $U$ of $t$ such that

$$
\left|[f(\sigma(t))-f(s)]-f^{\Delta}(t)[\sigma(t)-s]\right| \leq \varepsilon|\sigma(t)-s|, \forall s \in U,
$$

is called the delta derivative of $f$ at $t$.

The function $f^{\Delta}: \mathbb{T}^{k} \rightarrow \mathbb{R}$ is called the delta derivative of $f$ on $\mathbb{T}^{\kappa}$.

We say that $f$ is delta differentiable on $\mathbb{T}^{\kappa}$, if $f^{\Delta}(t)$ exists for all $t \in \mathbb{T}^{\kappa}$.

Definition 3. Let $f: \mathbb{T} \rightarrow \mathbb{R}$ and $t \in \mathbb{T}_{k}$. Then the number $f^{\nabla}(t)$ (when it exists), with the property that, for any $\varepsilon>0$, there exists a neighborhood $U$ of $t$ such that

$$
\left|[f(\rho(t))-f(s)]-f^{\nabla}(t)[\rho(t)-s]\right| \leq \varepsilon|\rho(t)-s|, \forall s \in U,
$$

is called the nabla derivative of $f$ at $t$.

The function $f^{\nabla}: \mathbb{T}_{k} \rightarrow \mathbb{R}$ is called the nabla derivative of $f$ on $\mathbb{T}_{\kappa}$.

We say that $f$ is nabla differentiable on $\mathbb{T}_{\kappa}$, if $f^{\nabla}(t)$ exists for all $t \in \mathbb{T}_{\kappa}$.

Remark 1 . If $\mathbb{T}=\mathbb{R}$, then $f: \mathbb{R} \rightarrow \mathbb{R}$ is both delta differentiable and nabla differentiable at $t \in \mathbb{R}$ iff

$$
f^{\Delta}(t)=f^{\nabla}(t)=\lim _{s \rightarrow t} \frac{f(t)-f(s)}{t-s}=f^{\prime}(t),
$$

i.e. $f$ is differentiable in the ordinary sense at $t$.

If $\mathbb{T}=\mathbb{Z}$, then $f: \mathbb{Z} \rightarrow \mathbb{R}$ is always delta differentiable and nabla differentiable on $\mathbb{Z}$ and 


$$
\begin{aligned}
& f^{\Delta}(t)=\frac{f(\sigma(t))-f(t)}{\mu(t)}=f(t+1)-f(t), \\
& f^{\nabla}(t)=\frac{f(t)-f(\rho(t))}{\nu(t)}=f(t)-f(t-1),
\end{aligned}
$$

for all $t \in \mathbb{Z}$.

Proposition 1. [12]

i) Assume that $f: \mathbb{T} \rightarrow \mathbb{R}$ is delta differentiable on $\mathbb{T}^{k}$. Then $f$ is nabla differentiable at $t$ and

$$
f^{\nabla}(t)=f^{\Delta}(\rho(t))
$$

for $t \in \mathbb{T}_{k}$ such that $\sigma(\rho(t))=t$. If, in addition, $f^{\Delta}$ is continuous on $\mathbb{T}^{k}$, then $f$ is nabla differentiable at $t$ and (1) holds for any $t \in \mathbb{T}_{k}$.

ii) Assume that $f: \mathbb{T} \rightarrow \mathbb{R}$ is nabla differentiable on $\mathbb{T}_{k}$. Then $f$ is delta differentiable at $t$ and

$$
f^{\Delta}(t)=f^{\nabla}(\sigma(t))
$$

for $t \in \mathbb{T}^{k}$ such that $\rho(\sigma(t))=t$. If, in addition, $f^{\nabla}$ is continuous on $\mathbb{T}_{k}$, then $f$ is delta differentiable at $t$ and (2) holds for any $t \in \mathbb{T}^{k}$.

Remark 2. If $t \in \mathbb{T}^{\kappa}$ satisfies $\rho(t)=t<\sigma(t)$, then the forward jump operator $\sigma$ is not delta differentiable at $t$.

Remark 3. A function $f: \mathbb{T} \rightarrow \mathbb{R}$ is called regulated if its right-side limits exist (finite) at all right-dense points in $\mathbb{T}$ and its left-side limits exist (finite) at all left-dense points in $\mathbb{T}$.

Definition 4. A function $f: \mathbb{T} \rightarrow \mathbb{R}$ is called $\mathrm{rd}$-continuous if it is continuous at the right-dense points in $\mathbb{T}$ and its left-sided limits exist at all left-dense points in $\mathbb{T}$.

The set of all rd-continuous functions is denoted by $\mathcal{C}_{r d}$. It may be shown [7] (Theorem 1.60) that

- $f$ is continuous $\Rightarrow f$ is rd-continuous $\Rightarrow f$ is regulated

- $\sigma$ is rd-continuous.

\section{External dynamical equivalence}

Let $\mathbb{T}$ be a homogeneous time scale. Consider a system $\Sigma$ of the following form:

$$
\begin{array}{r}
x^{\Delta}(t)=f(x(t), u(t)) \\
y(t)=h(x(t))
\end{array}
$$


where $t \in \mathbb{T}, x(t) \in \mathbb{R}^{n}, y(t) \in \mathbb{R}^{p}$ and $u(t) \in \mathbb{R}^{m}$. We shall assume that $f$ and $h$ are analytic, and that control $u$ may be infinitely many times differentiated as a function on the time scale $\mathbb{T}$.

By a trajectory of the system $\Sigma$ we will mean any triple of functions $(y(\cdot), x(\cdot), u(\cdot))$ defined on some interval $[a, b)$ that satisfies the equations of $\Sigma$. We assume that $a \in \mathbb{T}, b \in \mathbb{T}$ or $b=\infty$ and $[a, b)$ contains infinitely many points. The pair $(x(\cdot), u(\cdot))$ is an inner trajectory and the pair $(y(\cdot), u(\cdot))$ is an external trajectory of $\Sigma$. The set of all inner (external) trajectories of the system $\Sigma$ forms the inner (external) behavior of the system. It will be denoted by $B^{i}(\Sigma)$ (respectively by $B^{e}(\Sigma)$ ).

By $J(r)$ we will denote the space of all infinite sequences $S=\left(s^{(i)}\right)_{i \geq 0}, s^{(i)} \in$ $\mathbb{R}^{r}$. If $z: \mathbb{T} \rightarrow \mathbb{R}^{r}$ has infinitely many delta-derivatives, the map $Z: \mathbb{T} \rightarrow J(r)$ defined by $Z(t)=\left(z(t), z^{\Delta}(t), \ldots\right)$ is called the (infinite) jet of $z$. We shall consider real maps defined on $J(r)$. We assume that each such map $\varphi$ depends only on a finite number of elements of the sequence $S$ (but the number of these elements depends on the given map $\varphi$ ). In this case we say that the function $\varphi$ is finitely presented. A map $\phi: J(r) \rightarrow \mathbb{R}^{\tilde{r}}$ is finitely presented if all its components have this property.

Let us consider two systems on the time scale $\mathbb{T}$ :

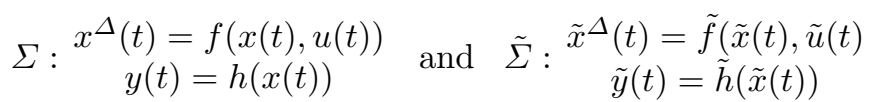

where $x(t) \in \mathbb{R}^{n}, \tilde{x}(t) \in \mathbb{R}^{\tilde{n}}, y(t) \in \mathbb{R}^{p}, \tilde{y}(t) \in \mathbb{R}^{\tilde{p}}, u(t), \tilde{u}(t) \in \mathbb{R}^{m}, t \in \mathbb{T}$.

Consider the following dynamical transformations:

$$
\begin{array}{ll}
y=\phi^{e}(\tilde{Y}, \tilde{U}), & u=\psi^{e}(\tilde{Y}, \tilde{U}) \\
\tilde{y}=\tilde{\phi}^{e}(Y, U), & \tilde{u}=\tilde{\psi}^{e}(Y, U)
\end{array}
$$

where $Y \in J(p), \tilde{Y} \in J(\tilde{p}), U, \tilde{U} \in J(m)$, and $\phi^{e}, \tilde{\phi}^{e}, \psi^{e}, \tilde{\psi}^{e}$ are finitely presented maps of the class $C^{\omega}$.

We say that $\Sigma$ and $\tilde{\Sigma}$ are externally dynamically equivalent, if there exist transformations (4) and (5) mutually inverse on systems' behaviors that induce the following relations between the external trajectories of both systems:

$$
\begin{aligned}
y(t) & =\phi^{e}\left(\tilde{y}(t), \tilde{y}^{\Delta}(t), \ldots, \tilde{y}^{\Delta^{k}}(t), \tilde{u}(t), \tilde{u}^{\Delta}(t), \ldots, \tilde{u}^{\Delta^{k}}(t)\right) \\
u(t) & =\psi^{e}\left(\tilde{y}(t), \tilde{y}^{\Delta}(t), \ldots, \tilde{y}^{\Delta^{k}}(t), \tilde{u}(t), \tilde{u}^{\Delta}(t), \ldots, \tilde{u}^{\Delta^{k}}(t)\right)
\end{aligned}
$$

for some integer $k \geq 0$, and similarly for $(\tilde{y}(t), \tilde{u}(t))$.

Example 4. Let $\Sigma$ be given by the equations:

$$
\begin{aligned}
x_{1}^{\Delta} & =\int_{0}^{1} \cosh \left(\sinh ^{-1}\left(x_{1}\right)+\mu h x_{2}\right) d h \cdot x_{2} \\
x_{2}^{\Delta} & =u \\
y & =x_{1} .
\end{aligned}
$$


Then $\Sigma$ is externally dynamically equivalent to the linear system $\tilde{\Sigma}$

$$
\tilde{x}^{\Delta}=\tilde{u}, \quad \tilde{y}=\tilde{x} .
$$

The equivalence transformations are given by

$$
\begin{aligned}
& \tilde{y}=\sinh ^{-1}(y) \\
& \tilde{u}=\frac{y^{\Delta}}{\cosh \left(\sinh ^{-1}(y)\right)}
\end{aligned}
$$

and

$$
\begin{aligned}
& y=\sinh (\tilde{y}) \\
& u=\tilde{u}^{\Delta} .
\end{aligned}
$$

For discrete-time systems the dynamical transformations (4) and (5) depend on forward differences, which in fact means dependence on future values of the output and the control. This is inconvenient from the practical point of view. However the same effect may by achieved by transformations that depend on past values of the output and the control (see [20]). This may be extended to homogeneous time scales with the following definition.

Assume that the trajectories of $\Sigma$ and $\tilde{\Sigma}$ are related by

$$
\begin{aligned}
& y(t)=\gamma^{e}\left(\tilde{y}(t), \tilde{y}^{\nabla}(t), \ldots, \tilde{y}^{\nabla^{l}}(t), \tilde{u}(t), \tilde{u}^{\nabla}(t), \ldots, \tilde{u}^{\nabla^{l}}(t)\right) \\
& u(t)=\eta^{e}\left(\tilde{y}(t), \tilde{y}^{\nabla}(t), \ldots, \tilde{y}^{\nabla^{l}}(t), \tilde{u}(t), \tilde{u}^{\nabla}(t), \ldots, \tilde{u}^{\nabla^{l}}(t)\right)
\end{aligned}
$$

for some integer $l \geq 0$ and

$$
\begin{aligned}
& \tilde{y}(t)=\tilde{\gamma}^{e}\left(y(t), y^{\nabla}(t), \ldots, y^{\nabla^{\tilde{l}}}(t), u(t), u^{\nabla}(t), \ldots, u^{\nabla^{\tilde{\tau}}}(t)\right) \\
& \tilde{u}(t)=\tilde{\eta}^{e}\left(y(t), y^{\nabla}(t), \ldots, y^{\nabla^{\tilde{l}}}(t), u(t), u^{\nabla}(t), \ldots, u^{\nabla^{\tilde{\tau}}}(t)\right)
\end{aligned}
$$

for some $\tilde{l}$, i.e. substituting an external trajectory of one system on the righthand side, we obtain an external trajectory of the other system on the lefthand side. We say that $\Sigma$ and $\tilde{\Sigma}$ are externally dynamically delay equivalent if applying transformations (7) to $(\tilde{y}, \tilde{u})$ and then transformations (8) to the resulting pair $(y, u)$, we finally obtain $(\tilde{y}, \tilde{u}) \circ \rho^{l+\tilde{l}}$ and the same holds the pair $(y, u)$ with the transformations applied in the reverse order. Thus the transformations (7) and (8) are mutually inverse on the external trajectories modulo the backward time shift $\rho_{\tilde{\Sigma}}^{l+\tilde{l}}$.

It can be shown that $\Sigma$ and $\tilde{\Sigma}$ are externally dynamically equivalent if and only if they are externally dynamically delay equivalent. This follows from the fact that on a homogeneous time scale we have $z^{\Delta^{r}}(t)=z^{\nabla^{r}}\left(\sigma^{r}(t)\right)$ and $z^{\nabla^{r}}(t)=z^{\Delta^{r}}\left(\rho^{r}(t)\right)$. Thus one can replace delta derivatives with nabla derivatives and vice versa, but some forward or backward shifts are involved in this operation. Moreover, the backward shift may be expressed with the aid 
of the nabla derivative. As the external dynamical equivalence is simpler from the mathematical point of view than the external delay dynamical equivalence, we shall concentrate on the former property in the rest of the paper.

External dynamical equivalence is an equivalence relation in the set of all control systems with output. It is a natural generalization of similar concepts for continuous-time and discrete-time systems.

We say that the system $\Sigma$ is externally dynamically linearizable if it is externally dynamically equivalent to a linear minimal one (i.e. controllable and observable).

To state a characterization of the external dynamical equivalence, we need the concept of function universe.

\section{Function universes}

Let $X, Y$ be sets. A partially defined function on $X$ with values in $Y$ is any map $\varphi: A \rightarrow Y$, where $A \subseteq X$ is called domain of $\varphi$ and denoted by $\operatorname{dom} \varphi$. If $\operatorname{dom} \varphi=\mathrm{X}$ then $\varphi$ is global. Let $Y_{X}$ be the set of all partially defined functions on $X$. One can extend any $\varphi \in Y_{X}$ to one defined on $X$ by assigning $\varphi(x)=\emptyset_{0}$ for $x \notin \operatorname{dom} \varphi$. We call $\emptyset_{0}$ the phantom. Now $\operatorname{dom} \varphi=\left\{\mathrm{x} \in \mathrm{X}: \varphi(\mathrm{x}) \neq \emptyset_{0}\right\}$. If $a \in Y, x \in X$ then we set $a_{X}(x):=a$.

Let $A_{n}$ denote the set of functions of class $C^{\omega}$, partially defined on open subsets in $\mathbb{R}^{n}$ with values in $\mathbb{R}$. In particular, $A_{0}$ can be identified with $\mathbb{R} \cup \emptyset_{0}$. The topology in $A_{0}$ can be defined as follows: a subset $B \subset A_{0}$ is open if $B=A_{0}$ or $B$ is an open subset in $\mathbb{R}$.

Functions $\varphi, \psi \in Y_{X}$ are matching, if they take on the same values on $\operatorname{dom} \varphi \cap \operatorname{dom} \psi$. Let us consider a set $M \subseteq Y_{X}$ of functions that are matching and define a function $\underline{M} \in Y_{X}: \underline{M}(x)=\emptyset_{0}$ if no function in $M$ is defined at $x$ and $\underline{M}(x)=\varphi(x)$ for any function $\varphi \in M$ defined at $x$. The process of constructing $\underline{M}$ is called amalgamation of the functions of $M$.

Let $\varphi_{1}, \ldots, \varphi_{k} \in \mathbb{R}_{X}$ and $F \in A_{k}$. Then $F \circ\left(\varphi_{1}, \ldots, \varphi_{k}\right)$ is a partially defined function on $X$ given by

$$
\left(F \circ\left(\varphi_{1}, \ldots, \varphi_{k}\right)\right)(x)=F\left(\varphi_{1}(x), \ldots, \varphi_{k}(x)\right)
$$

for $x \in X$. If $\varphi_{i}(x)=\emptyset_{0}$ or $\left(\varphi_{1}(x), \ldots, \varphi_{k}(x)\right) \notin \operatorname{domF}$ then $F\left(\varphi_{0}(x), \ldots, \varphi_{n}(x)\right)=$ $\emptyset_{0}$. The map

$$
\left(\varphi_{1}, \ldots, \varphi_{k}\right) \mapsto F \circ\left(\varphi_{1}, \ldots, \varphi_{k}\right)
$$

is called a substitution.

A set $\mathcal{U} \subseteq \mathbb{R}_{X}$ containing $0_{X}$ and closed under substitutions and amalgamation is called a function universe on the set $X$ [16]. A function subuniverse of the universe $\mathcal{U}$ is a subset $\hat{U} \subset \mathcal{U}$ that is a function universe on $X$. If $\mathcal{H} \subset \mathcal{U}$, then function subuniverse generated by $\mathcal{H}$ is the smallest subuniverse of $\mathcal{U}$ containing $\mathcal{H}[1]$. 
In a natural way a function universe $\mathcal{U}$ on $X$ induces a topology on $X$ : the open sets have the form $\operatorname{dom} \varphi: \varphi \in \mathcal{U}$.

Let $\mathcal{U}_{1}, \mathcal{U}_{2}$ be function universes on $X_{1}$ and $X_{2}$ respectively. A map $\tau$ : $\mathcal{U}_{1} \rightarrow \mathcal{U}_{2}$ is a homomorphism of function universes $\mathcal{U}_{1}$ and $\mathcal{U}_{2}$ if

1. $\tau\left(F \circ\left(\varphi_{1}, \ldots, \varphi_{k}\right)\right)=F\left(\tau \varphi_{1}, \ldots, \tau \varphi_{k}\right)$ for $\varphi_{1}, \ldots, \varphi_{k} \in \mathcal{U}_{1}, F \in A_{k}$

2. $\tau(\underline{M})=\tau(M)$ for any matching set $M \subset \mathcal{U}_{1}$

3. $\tau\left(0_{X_{1}}\right)=0_{X_{2}}$

If a homomorphism $\tau$ is a bijective map then it is an isomorphism of function universes.

\section{Conditions of equivalence}

We shall assume the following conditions on the dynamics of the system (3):

Condition D1. For every $x, y \in \mathbb{R}^{n}$ there is at most one $u$ that satisfies the equation

$$
y=f(x, u) .
$$

Condition D2. For any $x$ and $u$ the rank of the matrix

$$
\frac{\partial f}{\partial u}(x, u)
$$

is full (i.e. equal $m$ ).

Condition D3. The map

$$
\mathbb{R}^{n} \times \mathbb{R}^{m} \rightarrow \mathbb{R}^{n} \times \mathbb{R}^{n}:(x, u) \mapsto(x, f(x, u))
$$

is proper, i.e. the inverse image of a compact set in $\mathbb{R}^{n} \times \mathbb{R}^{n}$ is a compact set in $\mathbb{R}^{n} \times \mathbb{R}^{m}$.

Conditions D1-D3 were first introduced in $[14,15]$ to prove criteria of dynamical feedback equivalence for nonlinear continuous-time systems. Later there were used in [2] to show a similar result for nonlinear discrete-time systems, and recently, under the same assumptions, we have unified these characterizations stating the dynamical feedback equivalence criteria for nonlinear systems on homogeneous time scales [5].

The dynamical feedback equivalence concerns only the dynamical part of the system $\Sigma$, i.e. the equation

$$
x^{\Delta}(t)=f(x(t), u(t)) .
$$

Two systems are said to be dynamically feedback equivalent if they are externally dynamically equivalent after replacing their output functions by the identity maps (i.e. setting $y(t)=x(t)$ ). Then the dynamical transformations maps $\phi^{e}$ and $\psi^{e}$, denoted now by $\phi$ and $\psi$, depend rather on delta-derivatives of $x$ and $u$ and not of $y$ and $u$. 
Let $T$ be the map $\mathbb{R}^{n} \times J(m) \rightarrow J(n)$ defined by

$$
T\left(x_{0}, U\right):=X=\left(x^{(0)}, x^{(1)}, \ldots\right)
$$

where $(X, U)$ is the infinite jet at $t=0$ of the inner trajectory $(x, u)$ of $\Sigma$ that satisfies the initial condition $x(0)=x_{0}$. One can show that such $X$ is unique which means that $T$ is well defined. Moreover, Conditions D1 and D2 imply that $T$ is left invertible. Thus, if $X$ is the jet of the solution $x$ corresponding to a control $u$ (and the initial condition $x(0)=x_{0}=x^{(0)}(0)$ ), then the jet of $u$ can be expressed as $U=S(X)$, where components of $S$ are finitely presented and analytic. This means that the dynamical feedback equivalence is given by the equations

$$
\begin{aligned}
& x(t)=\phi\left(\tilde{x}(t), \tilde{x}^{\Delta}(t), \ldots, \tilde{x}^{\Delta^{k}}(t)\right) \\
& u(t)=\psi\left(\tilde{x}(t), \tilde{x}^{\Delta}(t), \ldots, \tilde{x}^{\Delta^{k}}(t), \tilde{u}(t), \tilde{u}^{\Delta}(t), \ldots, \tilde{u}^{\Delta^{k}}(t)\right)
\end{aligned}
$$

and similarly for $(\tilde{x}(t), \tilde{u}(t))$.

Let $A(n, m)$ denote the algebra of all finitely presented analytic functions $\varphi: \mathbb{R}^{n} \times J(m) \rightarrow \mathbb{R}$ and let $\Sigma$ be given by (3). Let us define the operator $\delta_{\Sigma}: A(n, m) \rightarrow A(n, m)$, associated with $\Sigma$, by

$$
\begin{aligned}
& \left(\delta_{\Sigma} \varphi\right)(x, U):= \\
& \int_{0}^{1} \frac{\partial \varphi}{\partial x}\left(x+h \mu f\left(x, u^{(0)}\right), U\right) d h \cdot f\left(x, u^{(0)}\right)+ \\
& \left.\sum_{i=0}^{\infty} \int_{0}^{1} \frac{\partial \varphi}{\partial u^{(i)}}\left(x, U+h \mu U_{1}\right)\right) d h \cdot u^{(i+1)}
\end{aligned}
$$

where $U_{1}=\left(u^{(1)}, u^{(2)}, \ldots\right)$. It is clear that this operator depends only on the dynamical part of $\Sigma$. It will be called the delta operator of the system $\Sigma$.

Remark 4. The delta operator has the following interpretation. Let $U(\cdot)=$ $\left(u(\cdot), u^{\Delta}(\cdot), \ldots\right)$ be the infinite jet of control $u$ and let $x(\cdot)$ be the solution of (3) corresponding to $u$ and the initial condition $x(0)=x_{0}$. Then the delta derivative at $t=0$ of $t \rightarrow \varphi(x(t), U(t))$ is equal to $\left(\delta_{\Sigma} \varphi\right)\left(x_{0}, U(0)\right)$. If $\mathbb{T}=\mathbb{R}$, then $\delta_{\Sigma}$ is a derivation of the algebra $A(n, m)$, i.e. it is linear and satisfies the Leibniz rule. This is not the case for other time scales. But because of the above interpretation we shall often call $\delta_{\Sigma} \varphi$ the $\delta_{\Sigma}$ derivative of $\varphi$.

The algebra $A(n, m)$ together with the operator $\delta_{\Sigma}$ is called the delta algebra of the system $\Sigma$ and denoted by $A_{\Sigma}$. A homomorphism of delta algebras $A_{\Sigma}$ and $A_{\tilde{\Sigma}}$ is a homomorphism $\tau: A(n, m) \rightarrow A(\tilde{n}, m)$ of algebras that satisfies the condition $\delta_{\tilde{\Sigma}} \circ \tau=\tau \circ \delta_{\Sigma}$. An isomorphism of the delta algebras $A_{\Sigma}$ and $A_{\tilde{\Sigma}}$ is a homomorphism that is a bijective map.

The main result of [5] says the following 
Theorem 1. Systems $\Sigma$ and $\tilde{\Sigma}$ are dynamically feedback equivalent iff their delta algebras $A_{\Sigma}$ and $A_{\tilde{\Sigma}}$ are isomorphic.

Now we are going to show a similar characterization of external dynamical equivalence for systems on homogeneous time scales.

Let $\mathcal{A}(n, m)$ denote the function universe of all partially defined and finitely presented analytic functions on $\mathbb{R}^{n} \times J(m)$. It is generated by the algebra $A(n, m)$. Observe that the operator $\delta_{\Sigma}$ can naturally be extended to $\mathcal{A}(n, m)$. For a system $\Sigma$ given by (3) we define the observation universe $\mathcal{U}_{\Sigma}$ to be the smallest subuniverse of $\mathcal{A}(n, m)$ containing the components $h_{i}$, $i=1, \ldots, r$, of the map $h$, the coordinate functions $u_{j}, j=1, \ldots, m$, and invariant under the action of $\delta_{\Sigma}$.

The observation universe $\mathcal{U}_{\Sigma}$ together with the operator $\delta_{\Sigma}$ is called the delta universe of the system $\Sigma$ (and will be denoted by the same symbol $\mathcal{U}_{\Sigma}$ ). A morphism of delta universes $\mathcal{U}_{\Sigma}$ and $\mathcal{U}_{\tilde{\Sigma}}$ is a morphism $\tau: \mathcal{U}_{\Sigma} \rightarrow \mathcal{U}_{\tilde{\Sigma}}$ of function universes that satisfies the condition

$$
\delta_{\tilde{\Sigma}} \circ \tau=\tau \circ \delta_{\Sigma} .
$$

An isomorphism of the delta universes $\mathcal{U}_{\Sigma}$ and $\mathcal{U}_{\tilde{\Sigma}}$ is a morphism that is a bijective map.

The system $\Sigma$ is called uniformly observable if every coordinate function $x_{i}, i=1, \ldots, n$, belongs to $\mathcal{U}_{\Sigma}$.

Remark 5. Uniform observability means that locally one can express coordinate functions as analytic functions of the output function $h$, the control $u$ and their $\delta_{\Sigma}$ 'derivatives'. This means that for every control function $u$, any two distinct initial states can be distinguished by observing the output. Moreover, we can recover the state from the output, the control and their derivatives. As in [20] we could assume specific conditions on $h$ and $f$ that guarantee uniform observability of the system. However we want to concentrate here on the problem of dynamical equivalence, so we put aside all the details concerning observability.

Proposition 2. The system $\Sigma$ is uniformly observable iff $\mathcal{U}_{\Sigma}=\mathcal{A}(n, m)$.

Proof. If $\Sigma$ is uniformly observable, then for every $i=1, \ldots, n, x_{i}$ belongs to $\mathcal{U}_{\Sigma}$. By definition, $\mathcal{U}_{\Sigma}$ contains also $u_{j}$ for $j=1, \ldots, m$ and all $\delta_{\Sigma}^{k} u_{j}=u_{j}^{(k)}$. Substituting $x_{i}, i=1, \ldots, n$, and $u_{j}^{(k)}, j=1, \ldots, m, k \geq 0$, into analytic partially defined functions we get all the elements of $\mathcal{A}(n, m)$. On the other hand, if $\mathcal{U}_{\Sigma}=\mathcal{A}(n, m)$, then every $x_{i}, i=1, \ldots, n$, belongs to $\mathcal{U}_{\Sigma}$.

Corollary 1. Assume that two systems $\Sigma$ and $\tilde{\Sigma}$ are uniformly observable. Then, $A_{\Sigma}$ and $A_{\tilde{\Sigma}}$ are isomorphic iff $\mathcal{U}_{\Sigma}$ and $\mathcal{U}_{\tilde{\Sigma}}$ are isomorphic.

Proof. From uniform observability we get $\mathcal{U}_{\Sigma}=\mathcal{A}(n, m)$ and $\mathcal{U}_{\tilde{\Sigma}}=\mathcal{A}(\tilde{n}, m)$. As $A(n, m)$ and $A(\tilde{n}, m)$ are the algebras of global functions of the universes 
$\mathcal{A}(n, m)$ and $\mathcal{A}(\tilde{n}, m)$, respectively, then the restriction of an isomorphism of $\mathcal{U}_{\Sigma}$ and $\mathcal{U}_{\tilde{\Sigma}}$ gives an isomorphism of $A_{\Sigma}$ and $A_{\tilde{\Sigma}}$. The other implication follows from the specific form of any isomorphism $\tau$ of $A_{\Sigma}$ and $A_{\tilde{\Sigma}}$ (see [5]). It is a pullback of a certain map, so it commutes with substitutions and amalgamations. Thus the isomorphism $\tau$ of $A_{\Sigma}$ and $A_{\tilde{\Sigma}}$ is also an isomorphism of the function universes $\mathcal{U}_{\Sigma}$ and $\mathcal{U}_{\tilde{\Sigma}}$. This also allows to show that the condition $\delta_{\tilde{\Sigma}} \circ \tau=\tau \circ \delta_{\Sigma}$ can be extended to the universes $\mathcal{U}_{\Sigma}$ and $\mathcal{U}_{\tilde{\Sigma}}$.

If $\theta$ is a real function on $\mathbb{R}^{n} \times J(m)$, then by $\Theta$ we denote its infinite deltajet: $\left(\theta, \delta_{\Sigma}(\theta), \delta_{\Sigma}^{2}(\theta), \ldots\right)$. This, in particular, concerns the coordinate functions $x_{i}$ and $u_{j}$ and their aggregations $x$ and $u$, whose delta-jets are denoted by $X$ and $U$.

Uniform observability of $\Sigma$ means that $x=\varrho(Y, U)$ for some finitely presented analytic map $\varrho$. Thus $X=R(Y, U)$, where $R$ is the infinite delta-jet of $\varrho$. This equation may be rewritten on the level of jets of functions of time: $X(t)=R(Y(t), U(t))$. On the other hand, from the relations $y=h(x)$, after applying the operator $\delta_{\Sigma}$, we get $Y=\Gamma(X, U)$, for some map $\Gamma$ with finitely presented components.

Proposition 3. Assume that two systems $\Sigma$ and $\tilde{\Sigma}$ are uniformly observable. Then, $\Sigma$ and $\tilde{\Sigma}$ are dynamically feedback equivalent iff they are externally dynamically equivalent.

Proof. Assume first that $\Sigma$ and $\tilde{\Sigma}$ are dynamically feedback equivalent. Thus there exist transformations $\phi, \psi, \tilde{\phi}$ and $\tilde{\psi}$ such that for an inner trajectory $(\tilde{x}, \tilde{u})$ of $\tilde{\Sigma}$ the equations

$$
\begin{aligned}
& x(t)=\phi(\tilde{X}(t)) \\
& u(t)=\psi(\tilde{X}(t), \tilde{U}(t))
\end{aligned}
$$

define an inner trajectory of $\Sigma$, and similarly for $(\tilde{x}(t), \tilde{u}(t))$. Thus

$$
\begin{aligned}
& y(t)=h(\phi(\tilde{X}(t))) \\
& u(t)=\psi(\tilde{X}(t), \tilde{U}(t))
\end{aligned}
$$

define an external trajectory of $\Sigma$. From uniform observability, $\tilde{X}=R(\tilde{Y}, \tilde{U})$. Thus

$$
\begin{aligned}
& y(t)=h(\phi(R(\tilde{Y}(t), \tilde{U}(t)))) \\
& u(t)=\psi(R(\tilde{Y}(t), \tilde{U}(t)), \tilde{U}(t))
\end{aligned}
$$

define the transformations $\phi^{e}$ and $\psi^{e}$. Similarly we get the transformations $\tilde{\phi}^{e}$ and $\tilde{\psi}^{e}$, which gives external dynamical equivalence of $\Sigma$ and $\tilde{\Sigma}$.

Now assume that $\Sigma$ and $\tilde{\Sigma}$ are externally dynamically equivalent. Thus external trajectories of both systems are related by equations 


$$
\begin{aligned}
& y(t)=\phi^{e}(\tilde{Y}(t), \tilde{U}(t)) \\
& u(t)=\psi^{e}(\tilde{Y}(t), \tilde{U}(t)) .
\end{aligned}
$$

They can be extended to jets

$$
\begin{aligned}
Y(t) & =\Phi^{e}(\tilde{Y}(t), \tilde{U}(t)) \\
U(t) & =\Psi^{e}(\tilde{Y}(t), \tilde{U}(t)) .
\end{aligned}
$$

Again from uniform observability, $x=\varrho(Y, U)$, so

$$
x(t)=\varrho\left(\Phi^{e}(\tilde{Y}(t), \tilde{U}(t)), \Psi^{e}(\tilde{Y}(t), \tilde{U}(t))\right) .
$$

However $Y=\Gamma(X, U)$ and $U=S(X)$, so we finally obtain

$$
\begin{aligned}
& x(t)=\phi(\tilde{X}(t)) \\
& u(t)=\psi(\tilde{X}(t), \tilde{U}(t)) .
\end{aligned}
$$

for some finitely presented maps $\phi$ and $\psi$. Similarly for $\tilde{x}$ and $\tilde{u}$.

We can state now the main result of this paper.

Theorem 2. Two uniformly observable systems $\Sigma$ and $\tilde{\Sigma}$ are externally $d y$ namically equivalent if and only if their delta universes $\mathcal{U}_{\Sigma}$ and $\mathcal{U}_{\tilde{\Sigma}}$ are isomorphic.

Proof. The theorem follows from Corollary 1 and Proposition 3.

Example 5. The concept of external dynamical equivalence of systems on time scales may be used to give a unified setting for external dynamical linearization. In [21] and [19] it was shown that a continuous-time (discrete-time) system is externally dynamically linearizable if and only if its differential output universe (difference output universe, respectively) is free. To have this result on an arbitrary homogeneous time scale, it is enough to replace differential and difference output universes by the delta universe of the system on the time scale.

\section{CONCLUSIONS AND FUTURE WORKS}

\subsection{Conclusions}

The theory of control systems on time scales allows to use a common language for continuous-time and discrete-time systems. The concept of external dynamical equivalence of control systems with output unifies the earlier notions introduced separately for continuous-time and discrete-time systems. It was shown that two uniformly observable systems on a time scale are externally dynamically equivalent if and only if their delta universes are isomorphic. This extends corresponding results for continuous-time and discrete-time systems, where differential and difference output universes were used instead of the delta universe. The language introduced here allows to study external dynamical linearizations for systems on time scales. 


\subsection{Future Works}

The main assumption on the systems in this paper is uniform observability. It was shown in [21] and [3] that for continuous-time and discrete-time systems the criterion of external dynamical equivalence holds without this assumption. Thus one can hope that also for systems on a time scale observability can be dropped. This, however, requires a thorough study of observability of systems on time scales. A more serious challenge is to extend the result to nonhomogeneous time scales. In this case the delta operator may depend on time and this requires a new language. Another interesting extension should lead to modification of the concept of dynamical equivalence by allowing a change of time or, more generally, time scale.

\section{References}

1. Bartosiewicz Z., Johnson J., Systems on universe spaces. Acta Applicandae Mathematicae, 34 (1994).

2. Bartosiewicz Z., Jakubczyk B., and Pawłuszewicz E., Dynamic feedback equivalence of nonlinear discrete-time systems. Proceedings of First International Symposium on Mathematical Models in Automation and Robotics, Sept. 1-3, 1994, Międzyzdroje, Poland, Tech. Univ. of Szczecin Press, Szczecin, 1994.

3. Bartosiewicz Z., and Pawłuszewicz E., External equivalence of unobservable discrete-time systems. Proceedings of NOLCOS'98, Enschede, Netherlands, July 1998.

4. Bartosiewicz Z., Pawłuszewicz E., Unification of continuous-time and discretetime systems: the linear case. Proceedings of Sixteenth International Symposium on Mathematical Theory of Networks and Systems (MTNS2004) Katholieke Universiteit Leuven, Belgium July 5-9, 2004, Leuven 2004.

5. Bartosiewicz Z., and Pawłuszewicz E., Dynamic feedback equivalence of nonlinear systems on time scales. Proceedings of 16th IFAC Congress, Prague, 2005 (CD-ROM)

6. Bartosiewicz Z., Pawłuszewicz E., Realizations of linear control systems on time scales, Control \& Cybernetics, vol. 35(2006) No4

7. Bohner M., PetersonA., Dynamic Equation on Time Scales, Birkhäuser, 2001

8. Charlet B., Levine J., Marino R., Sufficient conditions for dynamic state feedback linearization, SIAM J. Control and Optimization 29 (1991), pp. 38-57

9. Fausett L.V., Murty K.N., Controllability, observability and realizability criteria on time scale dynamical systems, Nonlinear Stud., vol.11, (2004), pp. 627-638

10. Fliess M., Lévine J., Martin Ph., Rouchon P., Sur les systemes non linéaires différentiellement plats. C. R. Acad. Sci. Paris Sér. I Math., 315, no. 5, (1992), pp. $619-624$

11. Goodwin G.C., Graebe S.F., Salgado M.E., Control System Design, Prentice Hall International, 2001

12. Gürses M., Guseinov G.Sh., Silindir B., Integrable equations on time scales, Journal of Mathematical Physics, 46, 113510 (2005) 
13. Hilger S., Ein Maßkettenkalkülmit Anwendung auf Zentrumsmannigfaltigkeiten, Ph.D. thesis, Universität Würzburg, 1988

14. Jakubczyk B., Remarks on equivalence and linearization of nonlinear systems, Proceedings of the 2nd IFAC NOLCOS Symposium, 1992, Bordeaux, France, pp. 393-397

15. Jakubczyk B., Dynamic feedback equivalence of nonlinear control systems, preprint, 1992

16. Johnson J., A generalized global differential calculus I, Cahiers Top. et Geom. Diff. XXVII(1986)

17. Martin Ph., Murray R.M., Rouchon P., Flat systems. Plenary Lectures and Mini-Courses, European Control Conference ECC'97, Bastin G., and Gevers M., Eds., Brussels, 1997

18. R.H. Middleton, G.C. Goodwin, Digital Control and Estimation: A Unified Approach, Englewood Cliffs, NJ: Prentice Hall, 1990

19. Pawłuszewicz E., Dynamic linearization of input-output discrete-time systems, in: Proceedings of the International Conference UKACC Control'96, Exeter, UK, 1996

20. Pawłuszewicz E., Bartosiewicz Z., External Dynamic Feedback Equivalence of Observable Discrete-time Control Systems, Proc. of Symposia in Pure Mathematics, vol.64, American Mathematical Society, Providence, Rhode Island, 1999

21. Pawłuszewicz E., Z. Bartosiewicz, Differential universes in external dynamic linearization, Proceedings of European Control Conference ECC'03, Cambridge, G.B. 2003

22. Pomet J.-B., A differential geometric setting for dynamic equivalence and dynamic linearization, in: Geometry in Nonlinear Control and Differential Inclusions, Banach Center Publications, vol.32, Institute of Mathematics, Polish Academy of Sciences, Warsaw, Poland 1995 